\title{
The Effects of Hypergravity on Xenopus Embryo Growth and Cardiac Hypertrophy
}

\author{
Bryce Joseph Duchman and Darrell Wiens \\ Department of Biology \\ University of Northern lowa \\ Cedar Falls, lowa 50614-0421 USA
}

Received: August 31, 2011 Accepted: January 21, 2012

\begin{abstract}
All life on earth has developed and evolved in a unity gravity (1G) environment. Any deviation below or above $1 \mathrm{G}$ could affect animal development, a period when much change occurs and sensitivity is high. We imposed simulated hypergravity through centrifugation and analyzed the effects on the overall body length and cardiac growth of Xenopus laevis embryos. We predicted that increased contractile force would be required from the heart to adequately circulate blood, dispersing nutrients, and that this would inhibit organism growth and possibly induce a state of hypertrophy. Embryos reaching gastrulation stage were exposed to a $7 \mathrm{G}$ or $1 \mathrm{G}$ (control) field via centrifugation for 96 hours. We then recorded behavior, mortality and took body length measurements. We found no significant differences in behavior or mortality, however, body length was significantly reduced by an average of $6.8 \%$ in the $7 \mathrm{G}$ group. We then fixed, embedded, sectioned and stained embryos in order to investigate the dimensions of cardiac tissue and of the cardiac region of the body using image analysis software. We found the $7 \mathrm{G}$ group had a significantly reduced average body cross-sectional area $(-18 \%)$ and yet a significantly larger ventricular cross-sectional area $(+36 \%)$ when compared to the $1 \mathrm{G}$ group. The average ratio of ventricle cross-sectional area to average body cross-sectional area was significantly higher in the $7 \mathrm{G}$ group when compared to the $1 \mathrm{G}$. From these data, we conclude that hypergravity has a significant inhibitory impact on the Xenopus laevis embryo growth and causes a significant increase in ventricle size.
\end{abstract}

\section{INTRODUCTION}

Gravity is omnipresent on the Earth's surface. Every known organism evolved under the constant force and mechanical stress of gravity. Because of the unbroken presence of gravity, many processes certainly evolved which are gravity dependent [1]. However, it is difficult to determine these dependencies under normal gravitational conditions. To better understand gravity-dependent processes, researchers perform experiments in which they alter the gravitational environment to observe its impact on biological processes. Gravity can be altered above (hypergravity) or below unit gravity (hypogravity or microgravity). Hypergravity and microgravity could potentially affect physiological processes differently, so both have been studied.

In this study we used Xenopus laevis, the robust and mainly aquatic African clawed frog. As with all amphibians, these frogs have a three-chambered heart with two atria and one ventricle. The myocardium of the single ventricle provides the force necessary to circulate blood and nutrients throughout the body. In other studies of altered gravity effects, several organisms, including mammals, birds, and amphibians, have been used [2-7]. Studies employing vertebrate animals can be compared to some extent because all vertebrates pass through similar developmental stages, develop a multichambered heart, and have evolved in the earth's $1 \mathrm{G}$ environment. Frogs are convenient experimental subjects, especially in terms of developmental biology, because they are small, experimentally accessible and have a well-known progression in embryonic, larval, and adult development. The stages designated by Nieuwkoop and Faber [8] for Xenopus laevis are based on morphology. Sixty-six stages chronicle the Xenopus laevis development from the fertilized one cell stage, through cleavage, 
gastrulation, neurulation, organogenesis, tadpole stages, and froglet stages, until adulthood is reached.

Furthermore, studies have shown that frogs experience changes in early development when subjected to altered gravity. When Xenopus laevis fertilized eggs were launched in a Maser- 6 sounding rocket to be subjected to microgravity in the few minutes following fertilization, they showed differences in morphology of the blastocoel compared to controls, but normal morphology resumed once back in a $1 G$ environment [9]. Other studies showed effects on the establishment of polarities and body axes, which were also regulated back to normal (10-12). However a more recent study [13] used a microgravity-simulating rotating wall vessel (bioreactor) to expose Xenopus laevis embryos to simulated microgravity for four days beginning at gastrulation (germ layers already formed). This study found significant abnormalities and malformations in head cartilages, structures derived from neural crest cells, which begin determination and migration following gastrulation.

To consider the opposite side of the gravity continuum, Kawakami et al [14] investigated frogs developing in hypergravity and reported severe effects on body structure. Their experiments imposed hypergravity at three different developmental stages: twenty minutes post insemination, at the two-cell stage, and at the gastrula stage. The Xenopus laevis frog embryos were exposed to $5 \mathrm{G}$ conditions by centrifugation until the control group hatched from the jelly coat. Those embryos subjected to hypergravity earlier in development displayed an increased number of fatalities and abnormalities, including microcephaly, microphthalmia, increased apoptosis in eye and brain tissues, and reduced body size. Another study by Remus and Wiens [15] examined development of Xenopus laevis in hypergravity ( $7 \mathrm{G}$ and $10 \mathrm{G}$ ), achieved by centrifugation for five days, beginning at the time of initiation of gastrulation (yolk plug stage). They also found evidence of significantly increased size and incidence of asymmetries in head cartilages. Debilitating malformations were not found more often in experimental embryos than control embryos, but embryos exposed to hypergravity were significantly shorter in overall body length.
These researchers hypothesized that reduced body size may be the result of reduced delivery of yolk nutrient material because the cardiovascular circulation was hindered by hypergravity, offering more resistance to cardiovascular circulation and potentially causing cardiac hypertrophy.

Not all processes are gravitydependent, however. Experimentation has shown that some physiological mechanisms are unaffected by gravity. For example, a centrifuge experiment on Xenopus A6 kidney cells demonstrated that the rate of cell proliferation did accelerate under hypergravity conditions (5G), but the increased gravity did not change the timeline for A6 cell differentiation [16]. Though studies such as this have found that not all processes are gravity-dependent, several studies have suggested that embryonic muscle development may be somewhat dependent on or influenced by gravity. One study suggested that skeletal and cardiac muscle became more organized and fibrils became denser when chick embryos developed in hypergravity (2G) compared to normal gravity [2]. The increase in cardiac cell organization was attributed to the possibility that hypergravity created conditions where stronger muscular activity was necessary for circulation of blood throughout the body. Another study [3] reported that exposure of neonatal rat cardiocytes to $2 \mathrm{G}$ centrifugation over 2 to 24 hours increased actin filament width and number. A separate study by Goldstein et al. [4] supported these claims, reporting that myofiber area increased significantly in adult rat papillary muscle when exposed to hypergravity (2G) for 14 days. This study also indicated that mitochondria from the cardiac muscle showed signs of fatigue typical of early stages of hypertrophy. All of these studies, however, took into account only the structural fibril formation of individual skeletal and/or cardiac cells. The gross structure or size of the embryonic heart organ after hypergravity exposure has not been investigated. Even the study that reported fatigued mitochondria [4] did not determine whether gross hypertrophy had occurred. Beyond effects on cardiac muscle, a 2004 study by Bozzo et al. [5] reported that hypergravity (2G) influenced rat skeletal muscle development when rats were exposed from conception to 100 days 


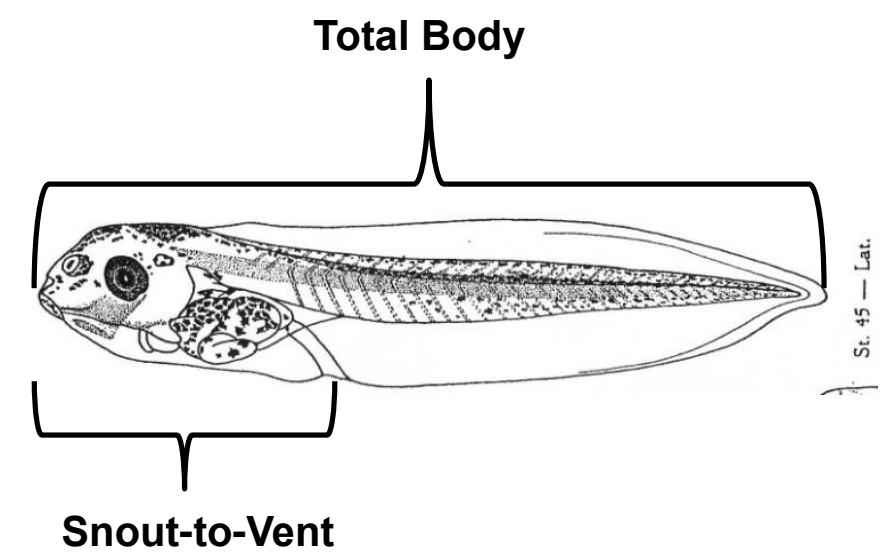

Figure 1. Stage 45 larva showing the two lengths that were measured.

old, based on muscle mass in relation to body mass and fiber diameter.

Cardiac hypertrophy is the increase in size of the cardiac muscle or myocardium. It is a common response to many cardiac stresses and pathologies, such as contractile protein dysfunction, cardiac arrhythmias, myocardial infarction, endocrine disorders, hypertension, and mechanical load. Initially, cardiac hypertrophy works to compensate in response to these stresses and pathologies and to sustain cardiac output. However, long periods of cardiac hypertrophy can lead to problems, such as heart fatigue, decreased compliance, and/or diastolic dysfunction, eventually resulting in overall weakness and decreased stroke volume [17, 18].

The first functioning unit in the developing vertebrate embryo is the circulatory system, and the heart within this system is the first functional organ. The system enables the delivery of nutrients to the developing body. It is plausible that cardiac hypertrophy could occur in embryos/larvae within the first four days of development when subjected to hypergravity conditions. In fact, a study published by Lwigale and others [6] reported that microgravity and hypergravity had a significant impact on the development of contractions in chick heart tissue explants. After exposure to simulated microgravity using a high aspect ratio vessel (HARV) bioreactor for 18 hours, the likelihood of the explants contracting in comparison to the control (1G exposure) was reduced by 21 $29 \%$. The effect of 18 hours of hypergravity exposure (1.4G \& 2.0G) by centrifugation was even more dramatic as the likelihood of the explants contracting in comparison to controls (1G exposure) was reduced by $84 \%$ in both experimental groups. In fact, none of the 47 chick cardiac explants developed contractions after exposure to more than negligible amounts of hypergravity [6]. If hypergravity really has such a significant impact on the development of cardiac muscle contraction, then cardiac stresses caused by hypergravity would be predicted, and could be expected to lead to the response of hypertrophy.

It is evident then, that hypergravity may influence gravity-dependent embryonic processes, and that cardiac hypertrophy and reduced body dimensions could result. Therefore, we analyzed the effects of hypergravity on the overall body length and cardiac growth of Xenopus laevis embryos exposed from gastrulation (approximately 911 hours post fertilization) to feeding tadpole stage (4-5 days).

\section{MATERIALS AND METHODS}

\section{a. Embryo Collection}

Xenopus laevis adult pairs were injected with human chorionic gonadotropin in the dorsal lymph sack to induce spawning according to established protocols [19, 20]. 
The embryos were sorted based on apparent fertilization and viability at midgastrulation (stages 10-12 according Nieuwkoop and Faber [8]). Only normal and undamaged embryos were selected. The jelly coats were removed by treatment in $2 \%$ cysteine in $20 \%$ Steinberg's solution (SS), $\mathrm{pH} 8.0$, for approximately 4 minutes. This was followed by one quick rinse using $20 \%$ SS, and then four five-minute rinses in $20 \%$ SS. The embryos were again sorted to remove the nonviable. The viable embryos were singly and randomly placed into Costar 24-well trays, each well containing $2 \mathrm{~mL}$ of freshly aerated $10 \%$ SS (pH 7.4). The trays were covered with unsealed lids to allow air exchange. Three trays were filled, a total of 72 embryos for each experimental run. Although several experimental runs were carried out, we encountered problems that disqualified some runs such as fungal contamination (eventually solved by stopping centrifugation long enough to replace the SS with freshly aerated SS), room temperature spikes that may have affected centrifuge temperature, and excess mortality (which did not affect centrifuged embryos more than stationary controls but was evident from the spawnings of some of the adults). We considered 50\% mortality to be excessive, and we found that mortality of embryos was commonly $30-40 \%$. We are reporting on one experiment that was successful in achieving all of the desired conditions.

\section{b. Exposure to Hypergravity or Earth Gravity}

To simulate the mechanical stress of hypergravity in the experimental group, two trays of 24 embryos each were placed within an Eppendorf $5810 \mathrm{R}$ centrifuge on the carriers of a swinging rotor (A-4-62). The trays were spun over the course of 96 hours at 210 RPM (generating $7 G$ at midtray). This endpoint was chosen because it is the approximate time of development before feeding begins (Stage 45, Nieuwkoop and Faber [8]). Thus, the neural crest cell derived head skeleton with its mandibles and ceratohyals would begin to function and yolk absorption would quickly attenuate. A $1 \mathrm{G}$-control tray of 24 embryos was secured to the top deck of the centrifuge to equalize the effect of extraneous vibrations. During centrifugation, the internal temperature of the centrifuge was monitored and adjusted to replicate the temperature of the room as closely as possible. During the second and third days of centrifugation, the centrifuge was stopped briefly to substitute the $10 \%$ SS with freshly aerated SS. During these short stoppage periods any dead embryos were recorded and discarded.

\section{c. Fixation and Embedment}

After the completion of the 96-hour experiment, the centrifuge was stopped and all three trays were removed from within or on top of the centrifuge apparatus. The embryos were observed to determine any differences in activity level or swim rate. This was done by observing time intervals between spurts of swimming and duration of swim time during such spurts. The observations were made within approximately thirty minutes after the end of rotation. After observing physical movement, the SS was pipetted out, and 1012 drops of freshly prepared 4\% paraformaldehyde were added to each well to fix the embryos. Measurements of total body and snout-to-vent lengths were made and recorded. The total body and snout-tovent lengths were determined with the assistance of binocular dissecting microscope and ruler. The span of measurements on a representative Stage 45 embryo [8] is shown in Figure 1. After fixation and measurements, the embryos were subjected to a dehydration process and then embedded in pure paraffin.

\section{d. Sectioning \& Counterstaining}

After embedment the embryos were sectioned at 4-6 $\mu \mathrm{m}$ onto water on Histogrip (Invitrogen) coated slides using a ReichertJung Histocut 820 microtome. The sections were then stained with eosin and hematoxylin and mounted under coverglass.

\section{e. Analysis of Sections}

The sections were viewed under an Olympus $\mathrm{CH} 30$ microscope and images were captured using a Hitachi HV-C20 3CCD video camera and Optimas (Media Cybernetics, Inc.) image analysis software. We measured the transverse cross sectional 

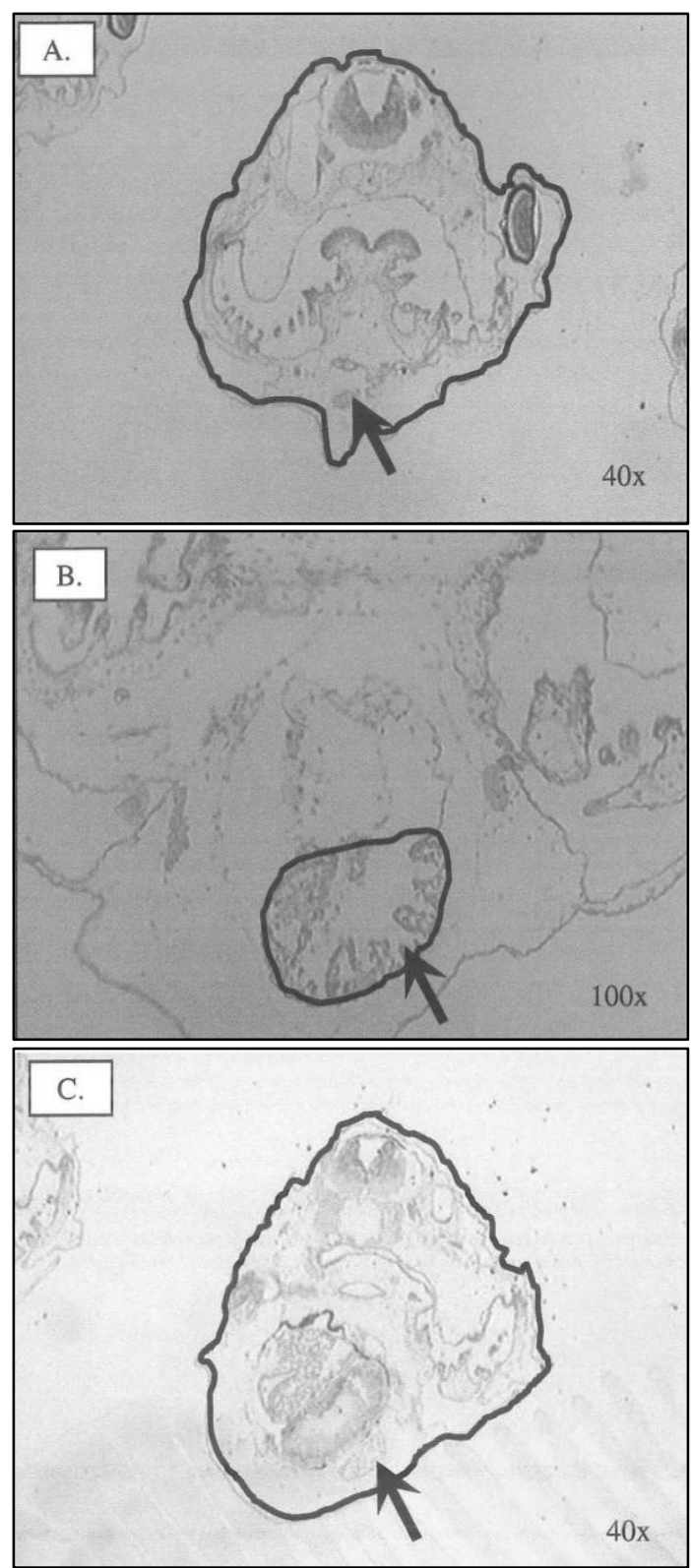

Figure 2. Cross-sections through five-day larvae showing areas circumscribed for measurement. A) Section through the most cranial end of the conotruncus (arrow). Black outline illustrates body area in this section. B) Section with the largest ventricular area. Arrow identifies the trabeculated ventricle tissue. C) Section with the most caudal ventricle portion. The arrow identifies the most caudal point of the ventricle (apex).

area of the ventricle where the heart area was largest in each embryo (Figure 2, B). We measured the transverse cross sectional body areas in two locations for each embryo at 40x magnification: where the cranial end of the conotruncus of the heart appeared, and again where the ventricle ended caudally, thus bracketing the heart (Figure 2). These two body areas were averaged to give the average cross sectional body area for the cardiac region. The average cardiac body areas were used to correct the ventricular areas for body size in the ratio of largest ventricular cross-section area/ average cardiac body area.

\section{f. Statistical Analysis of Data}

Once image measurements were made for all the embryos, we used SAS (Statistical Analysis System) software to execute Shapiro-Wilk normality tests on all data sets. SAS software was then used to perform Student's two-sample t-tests on the corresponding control and experimental data sets. SAS Software also was used to compute the correlation between ventricle area and average body area for 7G (experimental) and 1G (control) groups. The SAS correlation procedure calculated the Pearson product-moment correlation coefficient and significance probabilities.

\section{RESULTS}

\section{a. Mortality and Swimming Behavior}

When centrifugation was stopped briefly on the second day to replace the solution in each well with freshly aerated solution, dead embryos were removed and recorded. Mortality was higher in the $1 \mathrm{G}$ group with 10 out of 24 expiring (42\%), and 17 out of 48 expiring (35\%) in the $7 \mathrm{G}$ group, but this difference was not significant. In both groups, most mortality occurred within the first day. Upon observation at the end of 96 hours (observations were made within minutes of stopping the centrifuge), no noticeable differences in swim rate or activity levels were determined between the groups.

\section{b. Larvae Body Measurements}

We found that all of the data sets (including lengths, areas and ratios) were normally distributed according to Shapiro- 

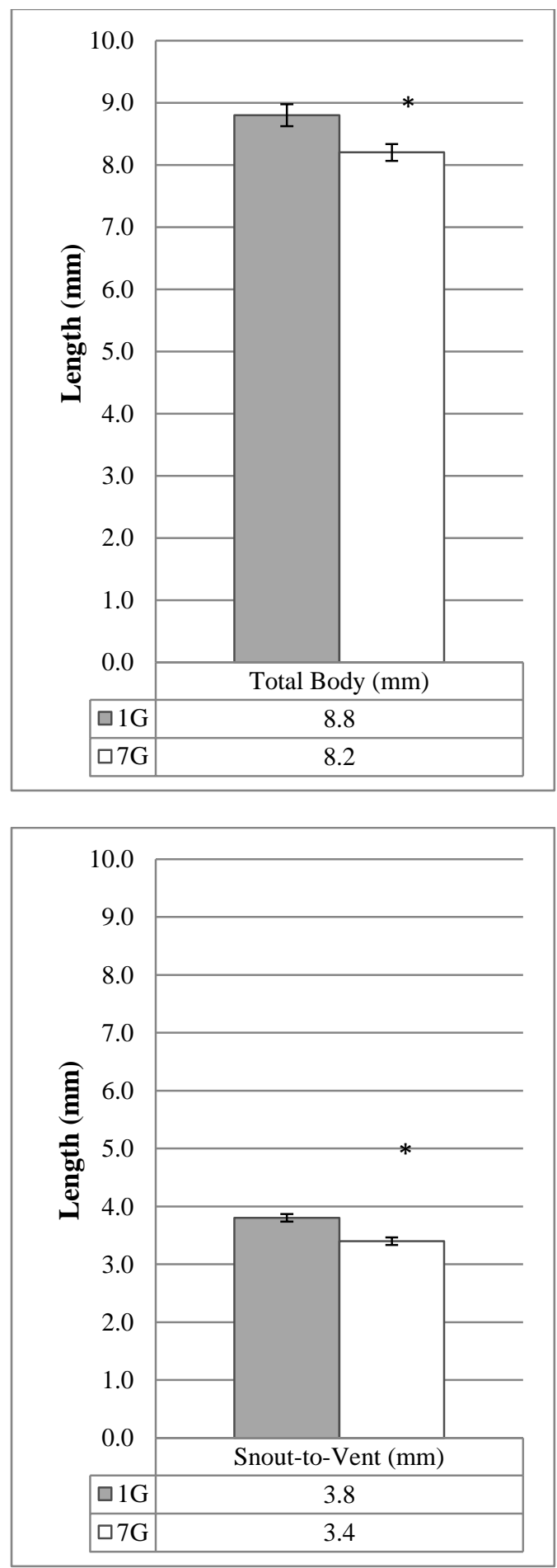

Figure 3. Mean whole body and snout-tovent lengths of five-day larvae that developed in $7 G$ or $1 G$. Both lengths at $7 G$ were significantly shorter than at $1 \mathrm{G}$ (control), $p \leq 0.05$.
Wilk normality tests. Therefore the data were all tested as indicated.

We discovered statistically significant differences between the $1 G$ and $7 \mathrm{G}$ groups in overall body and snout-to-vent lengths (Figure 3 ). The mean total body length of the $7 \mathrm{G}$ group was $0.6 \mathrm{~mm}$ shorter $(6.8 \%)$ than that of the $1 \mathrm{G}$ group. This difference was significant $(p<0.01)$. The mean snout-to-vent length was also shorter in the $7 \mathrm{G}$ group, reduced by $0.4 \mathrm{~mm}(11 \%)$, and this difference was also significant $(p<$ 0.001). For both measurements, the sample size (n) was 14 for the control group and 31 for the hypergravity group.

\section{c. Cross Sectional Ventricle and Body Areas in Cardiac Region}

We observed a statistically significant difference $(p<0.022, n=10$ for $1 G$ and 15 for $7 G$ ) between $1 G$ and $7 G$ groups in the cross sectional area of the ventricle where it was at its greatest in the sections (Figure 4). This area was enlarged $36 \%$ in the hypergravity group despite their having shorter bodies. In order to see whether these centrifuged larvae were also larger in the cardiac region of the body, we measured the cross-sectional area of the body. We found that in fact the body was significantly smaller $(p<0.047, n=10$ for the $1 \mathrm{G}$ and 16 for $7 \mathrm{G}$ group). The average cross sectional body area in the cardiac region was reduced almost $18 \%$ from 0.67 to $0.55 \mathrm{~mm}^{2}$ (Figure 4).

\section{d. Ratio of Largest Ventricle Area to Average Body Area}

Another way to consider these changes in heart and body size is to compare the ratio of the heart to the body in the cardiac region. Calculations revealed a statistically significant difference in the ratio of largest ventricle cross-sectional area to average body cross-sectional area (Figure $5)$. The average of the ratios was $60 \%$ larger for the $7 \mathrm{G}$ group, confirming that even though the body was shorter and narrower, the ventricle was greatly enlarged because of the exposure to hypergravity.
e. Correlation of Average Body Area to Largest Ventricle Area



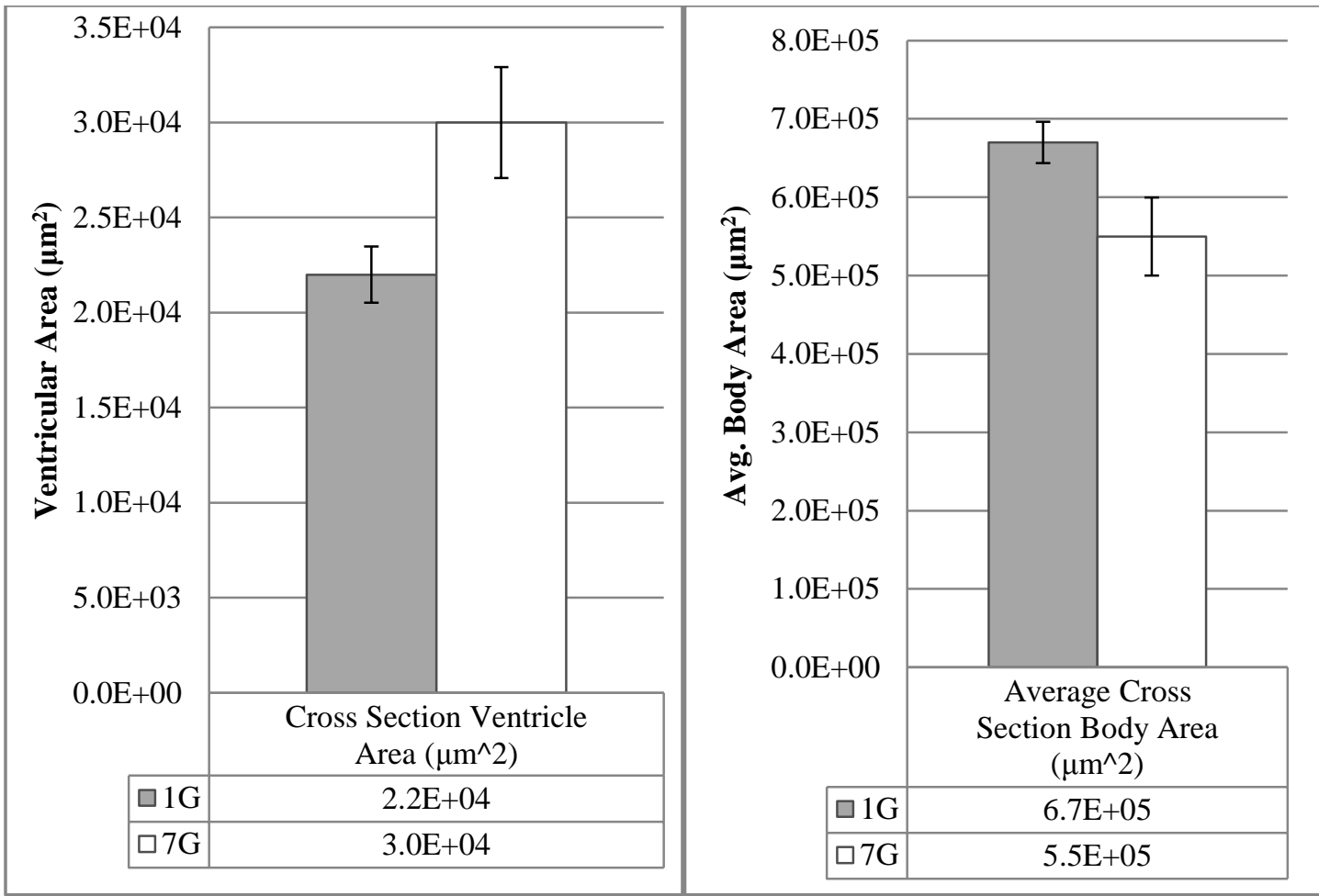

Figure 4. Mean cross-sectional areas of the body and ventricle in the heart region for $1 \mathrm{G}$ and $7 \mathrm{G}$ larvae. After 96 hours of development in $7 G$ the ventricles were significantly larger but the bodies significantly smaller in area. Note: *Significantly different from $1 G, p \leq 0.05$.

In order to determine whether crosssectional body and ventricular areas were correlated, the hypergravity and control groups were analyzed independently using the SAS correlation procedure, which calculated the Pearson product-moment correlation coefficient. This was found to be 0.48 for the $1 \mathrm{G}$ (control) group. Thus, $r^{2}=$ 0.23 , which was not significant $(p=0.166)$. The 7G group was found to have a correlation coefficient $(r)$ of 0.82 . Thus, $r^{2}=$ 0.68 , which was significant $(p<0.001)$. The correlation plot also revealed the much larger size of the ventricle in the body for the hypergravity group (Figure 6).

\section{DISCUSSION}

Although the effects of simulated hypergravity on amphibian development have been studied previously, there have been no studies specifically focusing on the gross structure or size of the embryonic heart after hypergravity exposure. Once the heart begins to circulate blood, it plays an important role in dispersing nutrients from the endodermal yolk mass within the gut to the rest of the developing body through the circulatory system. Increasing the force of gravity could resist the circulation of blood and nutrients to the growing body. In response to such potential cardiac stress, hypertrophy, a type of ventricular enlargement, can occur in an attempt to maintain cardiac output. Thus, the gross size of the amphibian embryonic ventricle would increase in response to hypergravity. Furthermore, if the dispersal of nutrients is hindered by hypergravity, then body dimensions might experience decreasing growth.

In this study, Xenopus laevis embryos were allowed to develop in unit gravity until mid-gastrulation, stage 10-12. Only then were the embryos exposed to simulated hypergravity via centrifugation. Therefore, the embryos avoided any effects hypergravity may have on early development and were able to establish blastomeres, signaling centers, and germ layers with normal determination and 


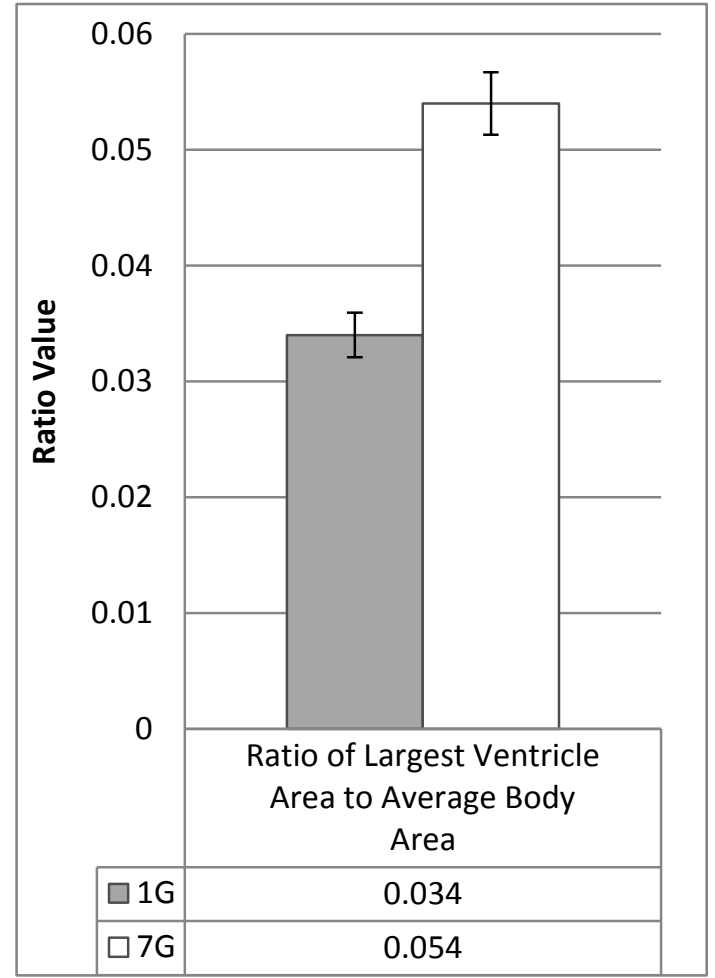

Figure 5. Mean ratio of largest ventricle area to average body area in Xenopus larvae that developed 96 hours at $1 \mathrm{G}$ or $7 \mathrm{G}$. Note: *Significantly greater than $1 \mathrm{G}$ control, $\mathrm{p}<0.001$.

proportion. They were then exposed to $7 G$ over a 4-day period (96 total hours), which takes the embryo through the stages of neurulation and organogenesis and up to the feeding tadpole stage (stage 45). The embryonic heart appears at stage 28-29 (35 hours of development) and begins to function at about stage 33 (45 hours of development). Thus the heart would be working against any extra resistance caused by hypergravity from its onset of function onward for at least two days.

The results are summarized in Table 1 . We found a statistically significant decrease in the total body length, an average of about $7 \%$, for embryos that developed in hypergravity conditions. Snout-to-vent lengths were found to be even more reduced (also significantly), about $10 \%$, for embryos that developed within the centrifuge, suggesting that hypergravity may have more effect on head and trunk than on tail (perhaps because of more arterioles and capillary beds). These findings paralleled the findings of Remus and Wiens [15], who found that embryos exposed to hypergravity were significantly shorter in overall body length, and even shorter at $10 \mathrm{G}$ than at $7 \mathrm{G}$.

We also found a statistically significant decrease in the average cross sectional body areas in the cardiac region for embryos that developed in hypergravity conditions. The decrease in body crosssectional area, total body length, and snoutto-vent length for embryos exposed to hypergravity supports the hypothesis that the dispersal of nutrients is hindered by hypergravity. This lack of dispersal would result in decreased body dimensions for the organism.

With regard to the heart dimensions, we found a statistically significant increase in largest ventricular area for embryos that developed in hypergravity conditions. This finding suggests that ventricle dimensions do increase in response to gravity during development. It should be noted that only transverse areas were determined, which take into account dorsal-ventral and left-right axis dimensions. Anterior-posterior axis dimensions of the heart or ventricle were not determined. Therefore, we cannot project an assessment of the total volume of ventricle tissue and cavity. Furthermore, increased ventricle dimensions do not necessarily equate to cardiac hypertrophy. The increased ventricle dimensions do suggest cardiac enlargement, but cardiac enlargement can occur due to cardiac dilation as well as cardiac hypertrophy. Cardiac dilation is known occur when an overabundance of blood enters or remains in a heart chamber because of a faulty heart valve. This causes the cavity to enlarge and the surrounding cardiac tissue to stretch; cardiac dilation differs from cardiac hypertrophy during which myocardial tissue actually thickens. We would not expect an abnormal amount of valve failure to occur during the experiment across the group of 31. However, we did not directly measure myocardium thickness because the wall trabeculation obscures the inside boundary. Therefore cardiac dilation remains a possible explanation of the results.

Since anterior-posterior dimensions were not determined, a ventricle volume to body volume ratio is not possible to calculate from our data. However, a 


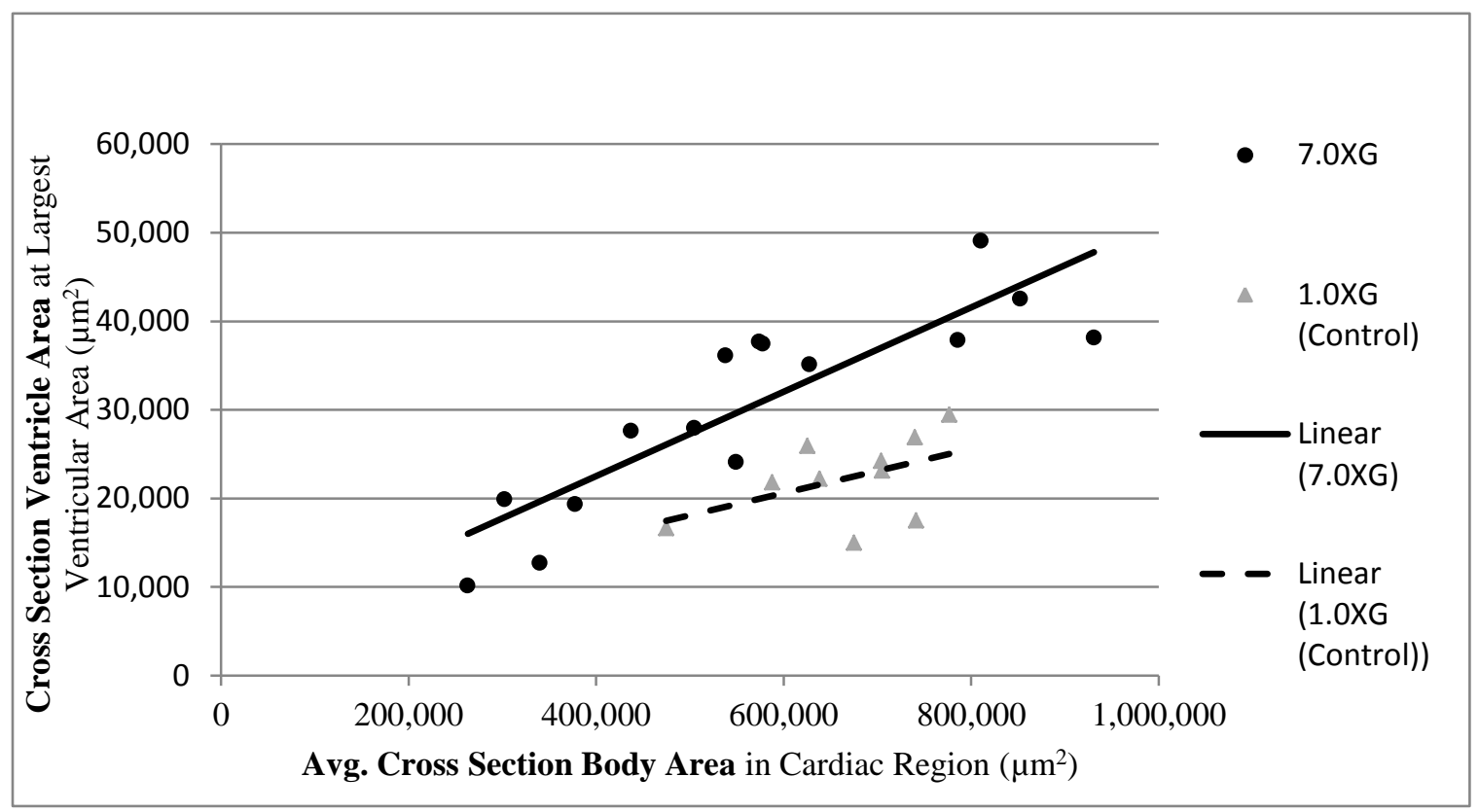

Figure 6. Correlation plot of ventricle cross-sectional area with average body cross-sectional area in the cardiac region. Although the correlation was not significant for $1 \mathrm{G}$ controls, it was significant for the $7 \mathrm{G}$ larvae, which had larger hearts.

ventricle area to average body area (within the cardiac region) ratio is possible to calculate. We did find a strong and statistically significant increase in the ventricle area to average body area ratio for embryos that developed in hypergravity. The ventricle area to average body area ratio gives a better value to assess the relationship between cardiac enlargement and body growth loss, for it allows the ventricle area values to be corrected for body size. The ratio gives a percentage of the average body area within the cardiac region that is taken up by the ventricle area. Therefore, a larger ratio value means the ventricle area is proportionally taking up more space within the body. We expected a larger ratio value for the $7 \mathrm{G}$ group because the ventricle size was already larger and the average body size already smaller. However, the strong statistical significance reveals that development in hypergravity really does appear to decrease the transverse cross-sectional body area and increase the transverse cross sectional ventricle area.

When each group was analyzed independently to determine the correlation between average body area and ventricle area variables, we found that the variables in the $1 \mathrm{G}$ group had a weak correlation and this correlation was not found to be statistically significant. However, we did find a fairly strong and statistically significant correlation between the variables for the $7 \mathrm{G}$ group $(n=15)$. This shows that the two dimensions altered by hypergravity are clearly related.

The data collected provides new insights about how hypergravity affects cardiac development. A logical next step to confirm cardiac hypertrophy in embryos developing in hypergravity would be to analyze the expression of cardiac hypertrophy marker peptides in control and hypergravity groups. Potential marker peptides include atrial natriuretic factor (ANF) and fibronectin. ANF is thought to be a cardiac hypertrophy modulator [21-23]. Thus ANF expression levels would theoretically increase during cardiac hypertrophy. Fibronectin is thought to play a role in modulating collagen orientation, thereby influencing myocardial compliance. Compliance is an important feature that is usually decreased by cardiac hypertrophy. Changes in expression levels may occur when compliance is affected by cardiac hypertrophy. Furthermore, fibronectin is known to accumulate in focal areas of 


\begin{tabular}{|c|c|c|c|}
\hline Data Set & $\begin{array}{c}1 \mathrm{G} \\
\text { (Mean } \pm \text { SD) }\end{array}$ & $\begin{array}{c}7 \mathrm{G} \\
\text { (Mean } \pm \text { SD) }\end{array}$ & $\mathrm{p}$ \\
\hline Total Embryo Length & $8.8 \pm 0.7 \mathrm{~mm}$ & $8.2^{*} \pm 0.8 \mathrm{~mm}$ & 0.011 \\
\hline Snout-to-Vent Length & $3.8 \pm 0.2 \mathrm{~mm}$ & $3.4^{*} \pm 0.4 \mathrm{~mm}$ & $<0.001$ \\
\hline $\begin{array}{c}\text { Cross Section Avg. Body } \\
\text { Area in Cardiac Region }\end{array}$ & $\begin{array}{c}0.67 \\
\pm 0.09 \mathrm{~mm}^{2}\end{array}$ & $\begin{array}{c}0.55^{*} \\
\pm 0.21 \mathrm{~mm}^{2}\end{array}$ & 0.047 \\
\hline $\begin{array}{c}\text { Cross Section Ventricle } \\
\text { Area at Largest Ventricular } \\
\text { Area }\end{array}$ & $\begin{array}{c}0.02 \\
\pm .005 \mathrm{~mm}^{2}\end{array}$ & $\begin{array}{c}0.03^{*} \\
\pm 0.011 \mathrm{~mm}^{2}\end{array}$ & 0.022 \\
\hline $\begin{array}{c}0.034 \\
\text { Ratio: Ventricle Area to } \\
\text { Avg. Body Area }\end{array}$ & $\begin{array}{c}0.054^{*} \\
\pm 0.011\end{array}$ & $<0.001$ \\
\hline
\end{tabular}

Table 1. Results Summary.

cardiac damage [24]. Thus, fibronectin expression levels may rise in response to cardiac damage occurring during the response of cardiac hypertrophy [22]. It is also reasonable to postulate that hypergravity differentially affects expression of genes that generally regulate cardiac and body growth. For example, Olson, et al [13] reported that exposure of early Xenopus embryos to microgravity reduced Hoxa2 expression. And Desprat et al [25] showed that the mechanical deformation caused by germ band extension in Drosophila embryos upregulates the expression of Twist in stomodeal cells and thereby causes midgut differentiation. Other effects of altered gravity or other type of mechanotransduction on gene expression are well known and reviewed by Ingber [26].

This study provides evidence that when Xenopus laevis embryos are exposed to simulated hypergravity during a period of yolk absorption and dispersion, the body dimensions are reduced and ventricle dimensions are enlarged. Although other explanations are possible, in particular an effect of hypergravity on gene expression, our results support the hypothesis that hypergravity increases the load on the cardiovascular system by resisting blood and nutrient circulation, which leads to decreased body dimensions and a cardiac hypertrophy response (or at least a cardiac enlargement response). Further studies will be needed to confirm and extend these findings.

\section{ACKNOWLEDGEMENTS}

This research was supported by undergraduate student research support from the Biology Department and the College of Natural Sciences at the University of Northern lowa. We thank Selena Losee, Kate Olson, Kerri Klemmensen and Erin O'kane for advice and technical assistance. We thank Dr. Steve O'Kane, Professor of Biology, and Jessica Moon, Director of University of Northern lowa Honors Program, for advice and support.

\section{REFERENCES}

1. Morey-Holton, E. (2003). The impact of gravity on life. Evolution on Planet Earth: Impact of the Physical Environment (1st ed., pp. 143-159). Toronto: Academic Press.

2. Sato, T., Shirai, W., Shioya, M., Kawashima, K., Endo, H., Sudoh, M. (1993). Enhancing effect of hypergravity on development of cardiac and skeletal 
muscles of chick embryos. Biological Sciences in Space, 7(1), 9-12.

3. Yang, F., Dai, Z., Tan, Y., \& Li, Y. (2010). Effects of altered gravity on the cytoskeleton of neonatal rat cardiocytes. Microgravity Science Technology, 22, 45-52.

4. Goldstein, M., Cheng, J., \& Schroeter, J. (1998). The effects of increased gravity and microgravity on cardiac morphology. Aviation, Space, and Environmental Medicine, 69(6), A12-6.

5. Bozzo, C., Stevens, L., Bouet, V., Montel, V., Picquet, F., Falempin, M., et al. (2004). Hypergravity from conception to adult stage: effects on contractile properties and skeletal muscle phenotype. The Journal of Experimental Biology, 207, 2793-2802.

6. Lwigale, P. Y., Thurmond, J. E., Norton, W. N., Spooner, B. S., \& Wiens, D. J. (2000). Simulated microgravity and hypergravity attenuate heart tissue development in Explant Culture. Cells Tissues Organs, 167, 171-183.

7. Davet, J., Fagette, S., Mani-Ponset, L., Bayard, B., Dumars, P., ReissBubenheim, D., et al. (1999). Cardiac atrial natriuretic peptide (ANP) in rat dams and fetuses developed in space (NIH-R1 and NIH-R2 Experiments). Life Sciences, 64(17), 1533-1541.

8. Nieuwkoop, P.D. and Faber J. (1967). Normal table of development of Xenopus laevis. (Daudin). North-Holland Publications, Amsterdam, 188 pages.

9. De Maziere, A., Gonzalez-Jurado, J., Reijnen, M., Narraway, J., \& Ubbels, G. (1998). Transient effects of microgravity on early embryos of Xenopus laevis. Advances in Space Research, 17(6/7), 219-223.

10. Gualandris-Parisot, L., Husson, D., Bautz, A., Durand, D., Kan, P., Aimar, C., Membre, H., Duprat, A.M. and Dournon, C. (2002). Effects of space environment on embryonic growth up to hatching of salamander eggs fertilized and developed during orbital flights. Biological Sciences in Space 16(1), 311.

11. Duprat A.M., Husson D. And Gualandris-Parisot L. (1998). Does gravity influence the early stages of the development of the nervous system in an amphibian? Brain Research Reviews 28(1-2), p.L19-24.

12. Souza K.A., Black S.D. And Wassersug R.J. (1995). Amphibian development in the virtual abence of gravity. Proceedings of the National Academy of Science USA92(6), p.1975-1978.

13. Olson, W., Wiens, D., Gaul, T., Rodriguez, M., \& Hauptmeier, C. (2010). Xenopus development from late gastrulation to feeding tadpole in simulated microgravity. The International Journal of Developmental Biology, 54, 167-174.

14. Kawakami, S., Kashiwagi, K., Furuno, N., Yamashita, M., \& Kashiwagi, A. (2006). Effects of hypergravity environments on amphibian development, gene expression, and apoptosis. Comparative Biochemistry and Physiology, 145, 65-72.

15. Remus, R., \& Wiens, D. (2008). The effects of hypergravity on the morphology of Xenopus embryos. American Journal of Undergraduate Research, 7(2), 19-26.

16. Tanaka, M., Asashima, M., \& Atomi, Y. (2003). Proliferation and differentiation of Xenopus A6 cells under hypergravity as revealed by time-lapse imaging. In Vitro Cellular \& Developmental Biology, 39, 71-79.

17. Frey, N., \& Olson, E. (2003). Cardiac hypertrophy: The good, the bad, and the ugly. Annual Review of Physiology, 65, 45-79.

18. Molkentin, J., Lu, J., Antos, C., Markham, B., Richardson, J., Robbins, J., et al. (1998). A calcineurindependent transcriptional pathway for cardiac hypertrophy. Cell, 93, 215-228.

19. Etheridge, A.L. and Richter, S.M.A. (1978). Xenopus laevis: Rearing and breeding the African Clawed Frog. 1978. NASCO, Publishing Agencies, Ft. Atkinson, WI.

20. Wu, M. and Gerhart, J. (1991) "Raising Xenopus in the laboratory." Methods in Cell Biology, 36, 3-18.

21. Ruskoaho H. (1992). Atrial natriuretic peptide: synthesis, release, and metabolism. Pharmacological Reviews, 44(4), 479-602.

22. Cao, L., \& Gardner, D. (1995). Natriuretic peptides inhibit DNA 
synthesis in cardiac fibroblasts. Hypertension, 25, 227-234.

23. Mori, T., Chen, Y., Feng, J., Hayashi, T., Oparil, S., \& Perry, G. (2004). Volume overload results in exaggerated cardiac hypertrophy in the atrial natriuretic peptide knockout mouse. Cardiovascular Research, 61, 771-779.

24. Mamuya, W.S., \& Brecher, P. (1992). Fibronectin expression in the normal and hypertrophic rat heart. The Journal of Clinical Investigation, 89, 392-401.
25. Desprat, N.., Supatto, W., Pouille, P.A., Beaurepaire, E., \& Farge, E. (2008). Tissue deformation modulates twist expression to determine anterior midgut differentiation in Drosophila embryos. Developmental Cell, 15, 470-477.

26. Ingber, D.E. (2006). Cellular mechanotransduction: putting all the pieces together again. FASEB Journal, 20, 811-827.

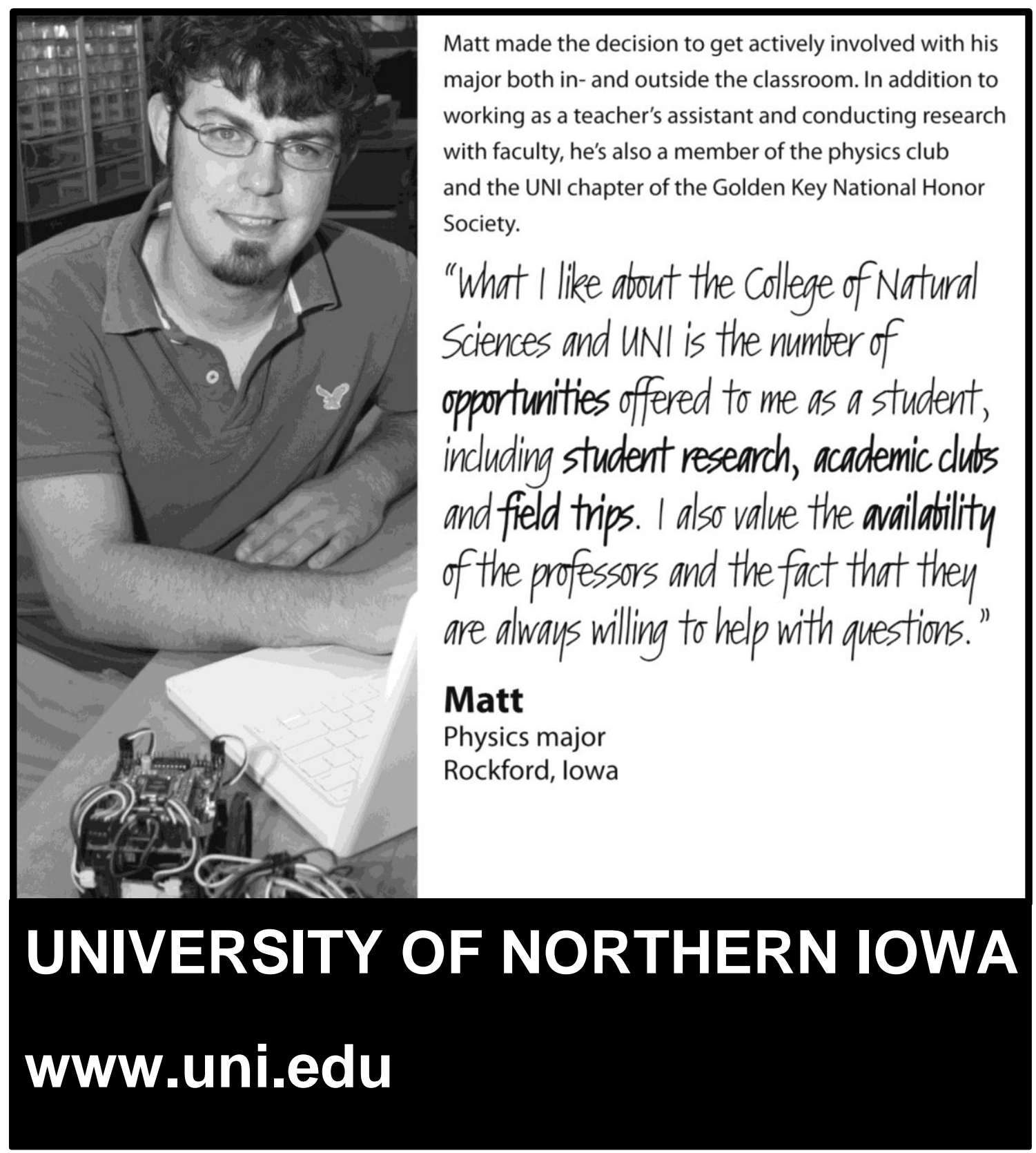

